coordinate only) CCs, and totally arbitrary WCs. Thus whereas KVs in the Einstein metrics are definite and only the temporal $\mathrm{RC}$ or $\mathrm{CC}$ becomes arbitrary, every direction $\xi^{\alpha}(\alpha=0, \ldots, 3)$ is a WC.

\title{
REFERENCES
}

Bokhari, A. H. (1990). International Journal of Theoretical Physics, 31, 2091.

Bokhari, A. H., and Qadir, A. (1990). Journal of Mathematical Physics, 31, 1463.

Bokhari, A. H., and Qadir, A. (1993). Joumal of Mathematical Physics, 34, 3543.

Bokhari, A. H., Amir, M. J., and Qadir, A. (1994). Journal of Mathematical Physics, 35, 3005.

Bokhari, A. H., Kashif, A. R., and Qadir, A. (n.d.). Curvature collineations of some spherically symmetric static spacetimes, accepted in Journal of Mathematical Physics, 1996.

Golab, S. (1972). Reports on Mathematical Physics B, 3, 21.

Kashif, A. R. (1995). M.Phil. dissertation, Quaid-i-Azam Ụniversity, Islamabad, Pakistan.

Katzin, G. H., and Levine, J. (1972). Colloquium Mathematicum, 26, 21.

Katzin, G. H., Levine, J., and Davis, H. R. (1969). Journal of Mathematical Physics, 10.617.

Qadir, A., and Ziad, M. (1993). In Proceedings of the Sixth Marcel Grossmann Meeting. T. Nakamura and H. Sato, eds., World Scientific, Singapore.

Ziad, M. (1990). Ph.D. thesis, Quaid-i-Azam University, Islamabad, Pakistan.

\section{Erratum: Weyl, Curvature, Ricci, and Metric Tensor Symmetries ${ }^{1}$}

\author{
Ashfaque H. Bokhari, ${ }^{2}$ Shahan Ahmad, ${ }^{2}$ and \\ Amjad Pervez ${ }^{2}$
}

We have found an error in one of the results in our paper. Our claim in Eq. (3) is not true. According to the correct version, all the Weyl tensor components in de Sitter/anti-de Sitter spacetimes are zero identically and therefore give arbitrary WCs and nol 10 as claimed in our paper. 